\title{
LOW THERMAL BUDGET SURFACE MICROMACHINING PROCESS FOR PIEZOELECTRIC MICROMACHINED ULTRASONIC TRANSDUCER ARRAYS WITH IN-SITU VACUUM SEALED CAVITIES
}

\author{
Qi Wang, Guo Lun Luo, Yuri Kusano, and David A. Horsley \\ University of California, Davis, USA
}

\begin{abstract}
This paper presents a low thermal budget surface micromachining process for piezoelectric micromachined ultrasonic transducer (PMUT) arrays with in-situ vacuum sealed cavities and high fill-factor. Low-temperature PECVD amorphous silicon (a-Si) and CMOS compatible aluminum nitride (AIN) are used in this process, making it possible to fabricate PMUT arrays on top of CMOS wafers directly at a low cost. The presented square PMUT array has a high fill-factor of $79 \%$ with a resonant frequency at 5.5 $\mathrm{MHz}$ in Fluorinert (FC-70, 3M), suitable for medical imaging applications. The transmitting surface pressure of this array is 2.93 $\mathrm{kPa} / \mathrm{V}, 1.25 \mathrm{x}$ higher than previously reported PMUT arrays for fingerprint sensors and the receiving sensitivity is $0.5 \mathrm{~V} / \mathrm{kPa}$ with a $10 \mathrm{~V} / \mathrm{pC}$ charge amplifier. The presented fabrication process also showed tunability of PMUT 3-dimension (3D) profile by controlling the AlN residual stress in the deposition process and the possibility to increase the dynamic responses. Meanwhile, the reliability of the PMUTs was tested for 21 hours continuous driving and was tested for seal quality when immersed in Fluorinert.
\end{abstract}

\section{INTRODUCTION}

Ultrasonic sensing for medical and biometric identification applications have been developed based on the recent research efforts with micromachined ultrasonic transducers (MUT) [1]-[3]. Both capacitive (CMUT) and piezoelectric micromachined ultrasonic transducers (PMUT) have been demonstrated as an alternative to current bulk piezoelectric ultrasonic transducers due to its small size, low cost for array fabrication, and CMOS integration compatibility. Cavities are essential for all MUT structures. Ideally, vacuum sealed cavities can eliminate the squeeze film damping during MUT operation and improve efficiency [4]. For CMUT, a small gap cavity is required to achieve high sensitivity [5]. Meanwhile, the small gap impedes a large displacement amplitude. For PMUT, the cavity gap can be made large. Currently available process to achieve this gap includes backside etching [6], utilizing cavity SOI wafers [7], wafer bonding [8] and epitaxial encapsulation [9]. Backside etching requires substrate thinning process and deep reactive etching (DRIE) from the backside of the wafer. The finished structure can be fragile due to the most released areas of the cavities. The cavities are exposed to the atmosphere and generate additional acoustic damping during the PMUT operation. Cavity SOI wafers can provide vacuum sealed cavities but are limited by a minimum spacing of $20 \mu \mathrm{m}$ which is large for highfrequency PMUT applications. Wafer bonding process was utilized in prior art fingerprint sensors. The process is expensive, unreliable and needs even larger bonding areas $\left(>400 \mathrm{~m}^{2}\right)$ limiting the fillfactor of high-frequency PMUT arrays [10]. Here we present a surface micromachining process to fabricate PMUT arrays with high fill-factor, high yield, vacuum sealed cavities, and reliable structures with high performance.

\section{FABRICATION PROCESS}

Figure 1 illustrates the four-mask PMUT surface micromachining process to achieve high fill-factor. The process

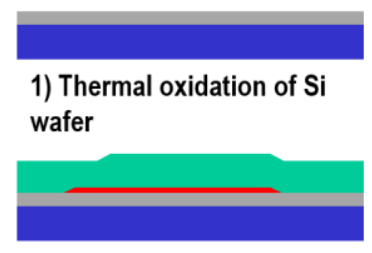

3) $1^{\text {st }}$ AIN deposition

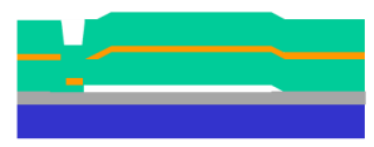

5) Mo and $2^{\text {nd }}$ AIN

deposition

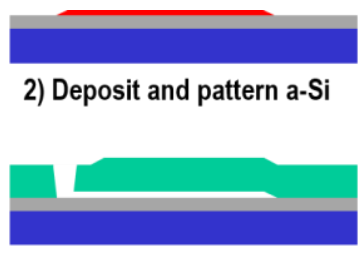

4) Release hole etching and $\mathrm{XeF} 2$ release

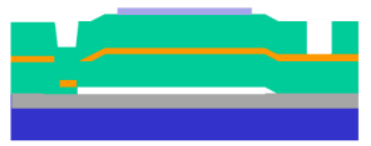

6) Al lift off for top electrode and AIN etch for bottom electrode

\section{$\mathrm{Si}$ \\ Figure 1: the surface micromachining process for high fill-factor} PMUT arrays.

starts by depositing a $500 \mathrm{~nm}$ thick silicon dioxide $\left(\mathrm{SiO}_{2}\right)$ as an insulation layer. Then a $500 \mathrm{~nm}$ thick PECVD a-Si was deposited and patterned with the $1^{\text {st }}$ mask to form the cavity sacrificial layer. The surface roughness of the $\mathrm{SiO}_{2}$ and a-Si is important for the subsequent aluminum nitride layer depositions. The minimum spacing between a-Si patterns defines the fill-factor and can be made as small as $5 \mu \mathrm{m}$ here, $4 \mathrm{x}$ smaller than the limits in commercially available cavity SOI wafers. The thickness of the a-Si layer defines the cavity depth. $500 \mathrm{~nm}$ thick is large enough for PMUT immersed applications like medical imaging or fingerprint sensors. A $500 \mathrm{~nm}$ AlN was firstly sputtered with an optimized recipe for achieving a lowest possible residual stress. The AIN layer was then patterned with the $2^{\text {nd }}$ mask to open the release holes. The diameter of the releasing holes can vary from $2 \mu \mathrm{m}$ to $10 \mu \mathrm{m}$ depends on cavity sizes. Then the a-Si sacrificial layer was removed in xenon difluoride $\left(\mathrm{XeF}_{2}\right)$ gas within 50 cycles. Each cycle lasts for 30 seconds and the $\mathrm{XeF}_{2}$ pressure is around 3.5 Torr. The minimum cavity spacing distance can be further reduced from $5 \mu \mathrm{m}$ if the anchors can sustain the released membranes. After the a-Si were completely removed, another $500 \mathrm{~nm}$ AlN, $150 \mathrm{~nm}$ molybdenum (Mo) and a $1 \mu \mathrm{m}$ AlN were sputtered in the same tool without breaking the vacuum. This deposition provided the in-situ vacuum sealed cavities due to the high vacuum in the chamber in the deposition process. The purpose of sputtering the bottom AlN layer in two steps is to provide a robust seal. The top $1 \mu \mathrm{m}$ AlN deposition recipe is optimized to achieve a highly c-axis oriented crystalline structure for good piezoelectric responses. Then the $3^{\text {rd }}$ mask is used to etch the top AlN layer and open the vias to bottom Mo electrode. In the last step, the top aluminum (Al) electrode with $300 \mathrm{~nm}$ thickness was evaporated and patterned with the 4th mask in a liftoff process.

\section{FABRICATION RESULTS}

The crystalline structure of the top AlN thin film is the key to Hilton Head Island, South Carolina, June 3-7, 2018 


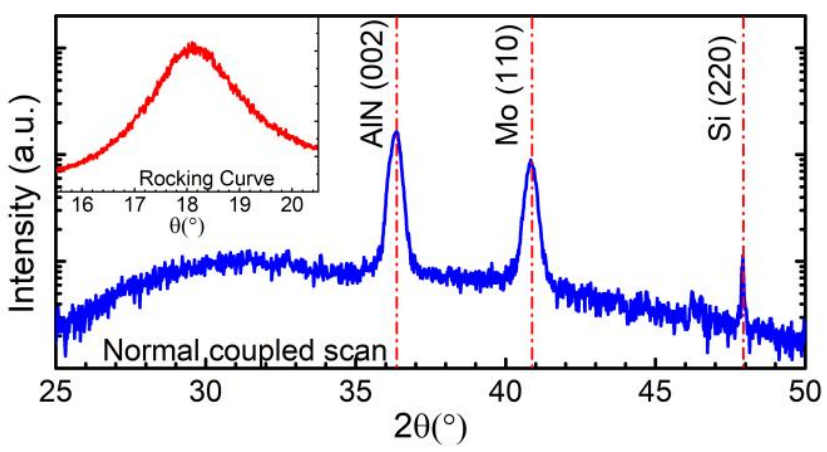

Figure 2: the $X$-ray diffraction normal coupled measurement results of the top AlN thin films; inset figure is the rocking curve measurement of the AlN (002) peak.

achieve good transmitting and receiving performance, and it was tested using X-ray diffraction (XRD, Siemens D5000 X-ray Diffractometer). Figure 2 shows the normal coupled $\theta-2 \theta$ scan results. Single AlN (002) peak is shown without other orientations, indicating that the film is c-axis oriented. Inset figure is the rocking curve measurement results at AlN (002) peak. The full-width-halfmaximum (FWHM) angle is $1.9^{\circ}$, indicating the AlN thin film is well oriented and predicting a good piezoelectric property.

This fabrication process allows us to create a 3D PMUT profile with AlN residual stress engineering. The same AlN sputtering recipe was used to deposit the bottom $1 \mu \mathrm{m}$ AlN thin film with a target of lowest residual stress on two wafers. Then different AlN sputtering recipes were used to deposit the top $1 \mu \mathrm{m}$ AlN thin films with different residual stresses. Therefore, different stress gradients were created on the top AlN in two wafers. The static deformation of the PMUTs was measured using a confocal microscope (Olympus LEXT OLS400) and the results are shown in Figure 3. Figure 3(a) indicates a convex PMUT profile with top AlN thin film to have more compressive stress than the bottom AlN thin film. Figure 3(b) shows the opposite results. A 2D symmetric finite element model (FEM) was created in COMSOL to study the effect of top AlN residual stresses. We assume that the bottom AlN thin film was stress-free and the top AlN thin films had residual stresses varying from $-100 \mathrm{MPa}$ to $+100 \mathrm{MPa}$. Figure 4(a) is showing the static
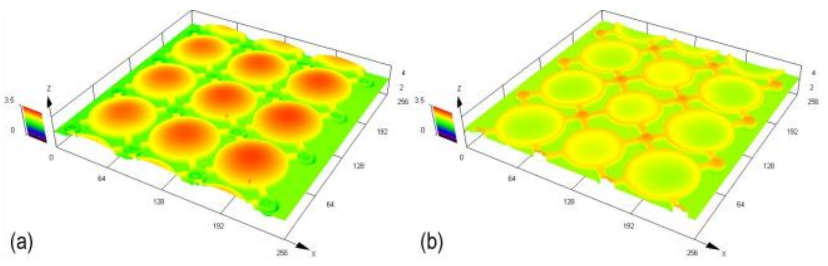

Figure 3: the confocal microscopic image of (a) convex and (b) concave PMUT array under different AIN deposition recipes.
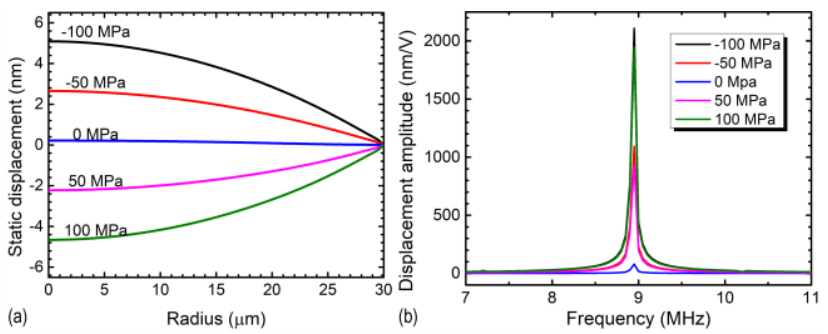

Figure 4: FEM simulation results of (a) symmetric static deformation profile and (b) dynamic displacement amplitude of PMUTs with a top AlN layer with a residual stress varying from -100 MPa to $100 \mathrm{MPa}$ and a bottom AlN layer with no residual stress.

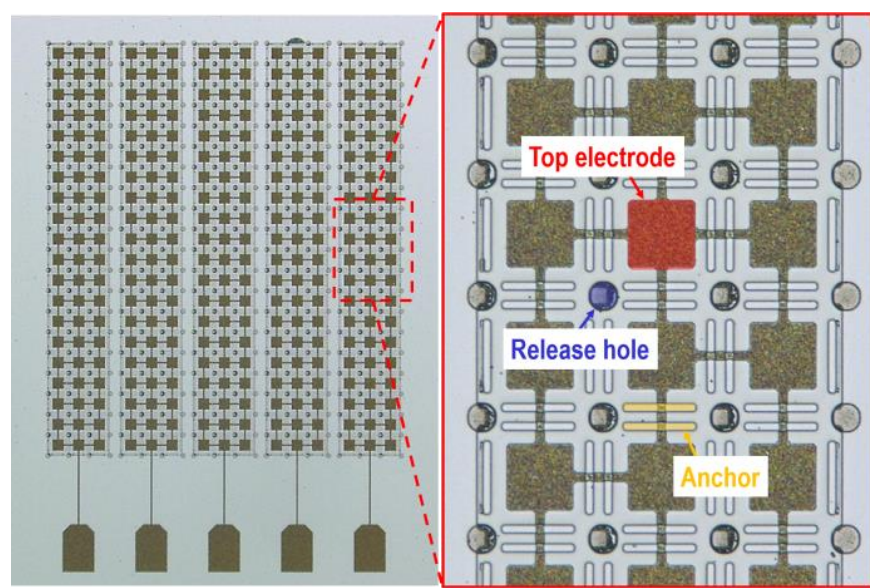

Figure 5: the optical microscopic image of the fabricated high fillfactor PMUT array.

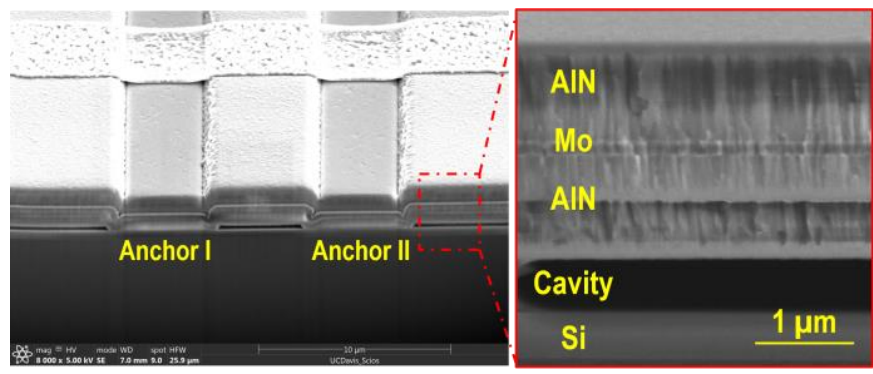

Figure 6: (left) the SEM image of the anchors and the well-sealed cavities; (right) the SEM image of the AlN/Mo/AlN layers with dense columnar AlN structures.

deformation caused by the stress variation. The results indicated that a tensile residual stress generated concave PMUT profile and a compressive residual stress generated convex PMUT profile as expected. The PMUT bending amplitude is proportional to the absolute value of the top AlN residual stress. The simulation results are consistent with the results shown in Figure 3. Figure 4(b) is showing the dynamic responses of the PMUT with top AlN thin films of varying residual stresses. The displacement amplitude increases with the increase of the absolute value of the residual stress. The signs of the residual stress does not influence the dynamic performance.

An optical microscopic image of the fabricated square PMUT array is shown in figure 5. The PMUT cavity is $50 \mu \mathrm{m}$ wide and the electrode is $35 \mu \mathrm{m}$ wide, targeting a $\sim 5 \mathrm{MHz}$ resonance in Fluorinert. The anchors are marked in the figure with the yellow color. The releasing hole of a diameter of $10 \mu \mathrm{m}$ is marked in the figure with blue color. The fill factor of this PMUT array is 79\%, the highest value for PMUT arrays to our best knowledge. In each element, there are 3 x 20 PMUT cells inside and are connected in parallel to increase the output charge/current. The number of the cells can be adjusted for electrical matchings with the transmitting or receiving circuitry. Five elements are fabricated here and the pitch between each element is $\sim 230 \mu \mathrm{m}$.

A focused ion beam (FIB, Scios Dual Beam SEM/FIB system) was used to open a trench of the approximate dimension of $50 \mu \mathrm{m}$ wide, $20 \mu \mathrm{m}$ long and $5 \mu \mathrm{m}$ deep. The scanning electron microscopic (SEM) image of the cross-section is shown in figure 6 . The two anchors and the cavities in between are shown in the figure. The AlN/Mo/AlN thin films stack is dense and continuous without any cracking. This shows that the cavity is properly sealed with the current fabrication process. A zoom-in figure of the SEM shows the 


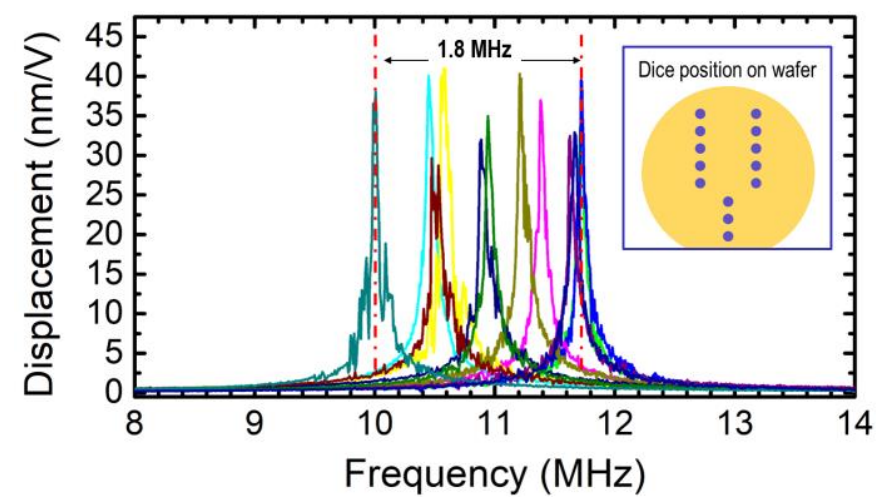

Figure 7: LDV measurement results of $1350 \mu \mathrm{m}$ wide square PMUTs across a wafer indicating a high displacement amplitude, and high yield. Inset figure shows the dice locations on the wafer.
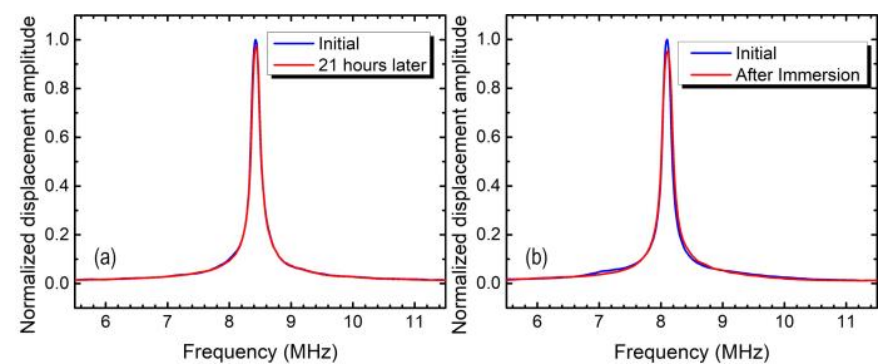

Figure 8: the displacement amplitude of a PMUT (a) before and after 21 hours continuous driving with $2.5 V_{p p}$ at $8.5 \mathrm{MHz}(b)$ before and after it is immersed in the fluid. No changes are observed indicating a good reliability and a good seal of the structure.

dense columnar structure of the AlN thin films with Mo electrode and the cavity. An interface of the bottom AlN can be seen in the figure and this is due to the different sputtering steps as mentioned earlier in the fabrication process.

\section{CHARACTERIZATIONS}

\section{Dynamic responses}

13 PMUT dices are selected across the wafer and the frequency responses are tested in the air with a laser Doppler vibrometer (LDV, OFV 512 and OFV 2700, Polytech) in conjunction with a network analyzer (E5601B, Agilent Technology). The results are plotted in figure 7. Inset figure is showing the positions of the 13 tested dices. The average displacement amplitude is $\sim 36 \mathrm{~nm} / \mathrm{V}$, the average resonant frequency is $\sim 11 \mathrm{MHz}$ and the average quality factor is 142. The displacement amplitude is 1.6x higher than circular $\mathrm{Sc}_{0.15} \mathrm{Al}_{0.15} \mathrm{~N}$ PMUT as reported in [7]. The variation of the resonant frequency is $\sim 1.8 \mathrm{MHz}$ and the probable reason for this change is the AlN residual stress variation across the wafer. Further efforts on better controlling the AlN stress across the wafer is needed to reduce the resonant frequency variation. The tested 13 dices all indicated high displacement amplitude is showing a high yield from this surface micromachining process. The differences in the resonant frequency in the air will not be influential when the devices are immersed in liquid and the small quality factor due to the large acoustic loading in liquid.

\section{Reliability}

A circular PMUT with $35 \mu \mathrm{m}$ radius was selected from the wafer and continuously driven in air at the resonant frequency, 8.5 $\mathrm{MHz}$, with $2.5 \mathrm{~V}_{\mathrm{pp}}$ sinusoid voltages for 21 hours. The displacement amplitude of the PMUT was continuously monitored via LDV. Figure 8 (a) shows the frequency response of the PMUT before

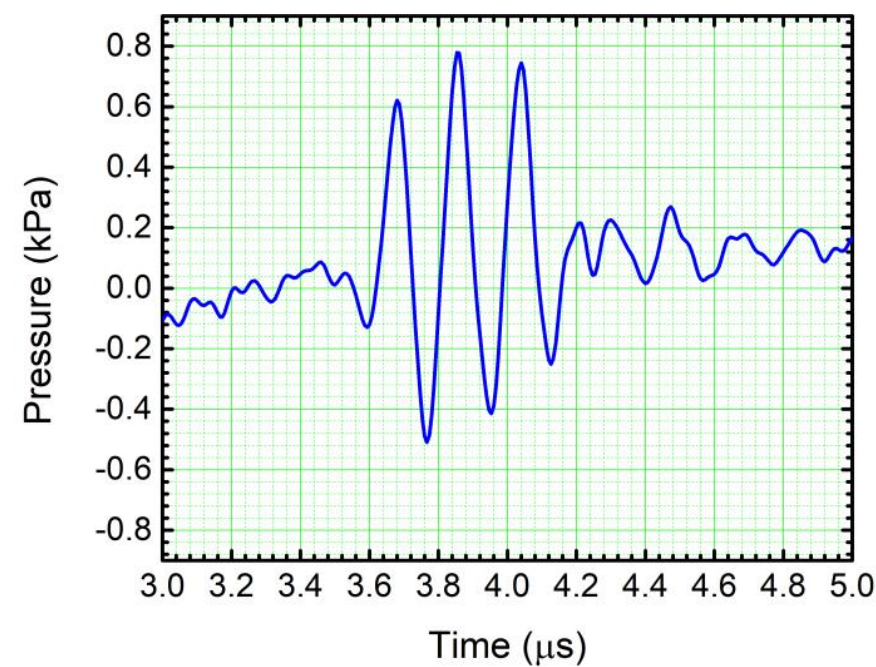

Figure 9: measured acoustic pressure in Fluorinert at $3 \mathrm{~mm}$ away from the PMUT surface.

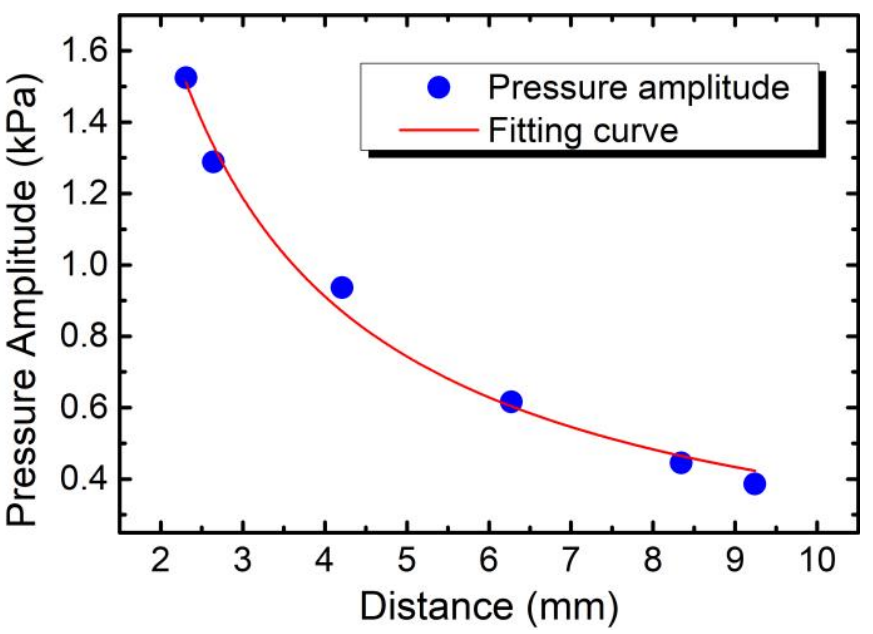

Figure 10: the measured pressure versus the measurement distance from the hydrophone to PMUTs surface and its fitting curve.

(blue) and after (red) this long time running tests. No obvious performance decay was observed from the figure. The testing results showed that the fabricated PMUTs are robust and can endure long working times without performance degradation. The negligible change in the frequency response also indicated that no structural damaged during this testing process.

Another circular PMUT with the same geometry was also tested for the cavity seal quality. The frequency response of this PMUT was first measured and the results are indicated in Figure 8(b) in blue. The PMUT dice was then immersed in Fluorinert for one hour. After the immersion, the dice was picked out and air dried to remove the surface attached Fluorinert liquid. In the end, the PMUT frequency response was measured again and the results are shown in the red line in figure 7(b). The frequency response before and after immersion overlapped and no obvious resonant frequency change or amplitude degradation was observed from this test. If there were leakage happened during this testing, the resonant frequency would be lower, and the quality factor would be smaller due to the added liquid damping induced by the liquid leakage. The results showed that the cavity is well sealed, and no leakage was found in this case.

\section{Pressure measurement}




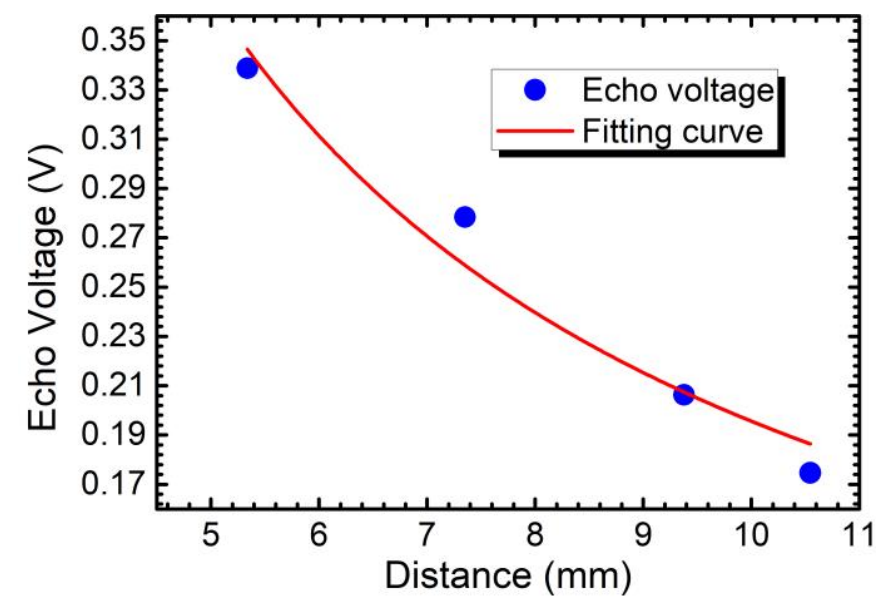

Figure 11: the measured echo output voltages versus the acoustic path and its fitting

One element of the square PMUT array was used to test its transmitting sensitivity. The PMUT element was immersed in Fluorinert and driven by a high voltage pulser (Microchip, HV7355DB1) with 3 unipolar pulses at $5.5 \mathrm{MHz}$ with $30 \mathrm{~V}_{\mathrm{pp}}$. A hydrophone (Precision acoustics) with $75 \mu \mathrm{m}$ diameter was used to measure the transmitted acoustic pressure on top of the PMUT surface. Figure 9 shows the measured acoustic pressure amplitude was $\sim 1.3 \mathrm{kPa}$ and it was measured $3.6 \mu$ s after the pulses were generated. The measured time corresponding to the relative distance between the hydrophone and the PMUT surface. The hydrophone was lifted gradually so that the output pressure at different heights were measured and results are shown in figure 10. The measured pressure amplitudes were fitted with the following equation, $p(x)=0.0058 x^{-0.92}$, where $x$ is the acoustic path distance. The Rayleigh distance of this element is $\sim 67.6 \mu \mathrm{m}$ and the output surface pressure is $\sim 2.93 \mathrm{kPa} / \mathrm{V}, 1.25 \mathrm{x}$ higher than reported in [11].

\section{Pulse-echo measurement}

The pulse-echo measurement was conducted with one element used as a transmitter and a neighbor element is used as a receiver. The same pulser was utilized in this measurement with the same condition as mentioned in pressure measurement. The receiving element was connected to a charge amplifier (FEMTO, HQA-15M$10 \mathrm{~T}$ ) with a gain of $10 \mathrm{~V} / \mathrm{pC}$. The output pressure propagated to the interface of the Fluroinert and air and then reflected. The PMUT receiving voltage from the echo pressure was amplified and shown on an oscilloscope. The Fluroinert height was adjusted so that the relationship between PMUT receiving voltage and acoustic path distance can be obtained. The results are plotted in figure 11 and are fitting results are $V(x)=0.0029 x^{-0.91}$. The receiving sensitivity can be obtained using $V(x) / p(x)$, which leads to $0.51 \mathrm{~V} / \mathrm{kPa}$. The imaging depth of this element is around $1 \mathrm{~cm}$ under $30 \mathrm{~V}_{\mathrm{pp}}$ pulses and it can be increased with large element sizes, higher transmitting voltages, and driving more elements with beam forming for real medical imaging applications.

\section{CONCLUSION}

Here, we presented a low cost, low thermal budget, CMOS compatible surface micromachining process with four-mask and insitu vacuum sealed cavities for fabricating high fill-factor PMUT arrays. The proposed fabrication process demonstrated PMUTs with high transmitting and receiving sensitivities, high yield, and high reliability. This fabrication process also enables us to tune the $3 \mathrm{D}$ geometry of the fabricated PMUT with either convex or concave structures without complicated etching steps as mentioned in [12] and improved the AlN piezoelectric properties. This fabrication process can be further improved with the potentials of using different sacrificial layer and elastic layer selections to create more robust, higher sensitivity PMUT structures and arrays.

\section{ACKNOWLEDGMENT}

The devices were fabricated in Berkeley Marvell Nanofabrication Laboratory. The authors thank Berkeley Sensor and Actuator Center (BSAC) industrial members for financial support.

\section{REFERENCES}

[1] Y. Lu et al., "Ultrasonic fingerprint sensor using a piezoelectric micromachined ultrasonic transducer array integrated with complementary metal oxide semiconductor electronics," Appl. Phys. Lett., vol. 106, no. 26, p. 263503, Jun. 2015.

[2] A. Caronti et al., "Capacitive micromachined ultrasonic transducer (CMUT) arrays for medical imaging," Microelectron. J., vol. 37, no. 8, pp. 770-777, Aug. 2006.

[3] F. L. Degertekin, "Microscale systems based on ultrasonic MEMS-CMOS integration," in 2017 19th International Conference on Solid-State Sensors, Actuators and Microsystems (TRANSDUCERS), 2017, pp. 397-401.

[4] Y. Lu and D. A. Horsley, "Modeling, Fabrication, and Characterization of Piezoelectric Micromachined Ultrasonic Transducer Arrays Based on Cavity SOI Wafers," $J$. Microelectromechanical Syst., vol. 24, no. 4, pp. 1142-1149, Aug. 2015.

[5] I. O. Wygant, et al., "Analytically calculating membrane displacement and the equivalent circuit model of a circular CMUT cell," in 2008 IEEE Ultrasonics Symposium, 2008, pp. 2111-2114.

[6] Benjamin Eovino, et al., "Broadband ring-shaped PMUTs based on acoustically induced resonance." in 2017 IEEE 30th International Conference on Micro Electro Mechanical Systems (MEMS), 2017, pp. 1184-1187.

[7] Q. Wang, et al., "Design, Fabrication, and Characterization of Scandium Aluminum Nitride-Based Piezoelectric Micromachined Ultrasonic Transducers," J. Microelectromechanical Syst., vol. 26, no. 5, pp. 1132-1139, Oct. 2017.

[8] J. M. Tsai, et al., "Versatile CMOS-MEMS integrated piezoelectric platform," in 2015 Transducers - 2015 18th International Conference on Solid-State Sensors, Actuators and Microsystems (TRANSDUCERS), 2015, pp. 2248-2251.

[9] Y. Chen, et al., "Robust Method of Fabricating Epitaxially Encapsulated MEMS Devices with Large Gaps," J. Microelectromechanical Syst., vol. 26, no. 6, pp. 1235-1243, Dec. 2017.

[10] Y. Lu et al., "Waveguide piezoelectric micromachined ultrasonic transducer array for short-range pulse-echo imaging," Appl. Phys. Lett., vol. 106, no. 19, p. 193506, May 2015.

[11] X. Jiang et al., "Monolithic 591x438 DPI ultrasonic fingerprint sensor," in 2016 IEEE 29th International Conference on Micro Electro Mechanical Systems (MEMS), 2016, pp. 107-110.

[12] S. Akhbari, et al., "Highly responsive curved aluminum nitride pMUT," in 2014 IEEE 27th International Conference on Micro Electro Mechanical Systems (MEMS), 2014, pp. 124127.

\section{CONTACT}

*Q. Wang, tel: +1-530-746-1632; qixwang@ucdavis.edu 